Z Gerontol Geriat 2017 · 50:655-656 https://doi.org/10.1007/s00391-017-1308-1

Online publiziert: 18 . September 2017

๑) Springer Medizin Verlag GmbH 2017

\section{Jürgen M. Bauer ${ }^{1} \cdot$ H. J. Heppner ${ }^{2}$}

'Geriatrisches Zentrum der Universität Heidelberg, Agaplesion Bethanien Krankenhaus Heidelberg Heidelberg, Deutschland

${ }^{2}$ HELIOS Klinikum Schwelm, Geriatrische Klinik und Tagesklinik, Universität Witten/Herdecke, Schwelm, Deutschland

\title{
50 Jahre und um manches weiser
}

50 Jahre Zeitschrift für Gerontologie und Geriatrie (ZGG) - ein schönes Jubiläum, aber auch Anlass für eine Bestandsaufnahme zur Entwicklung der Geriatrie in Deutschland, deren Spiegel die ZGG in vieler Hinsicht ist. Im zurückliegenden halben Jahrhundert hat sich die ZGG zur bedeutendsten deutschsprachigen Zeitschrift für die Themengebiete Gerontologie und Geriatrie entwickelt. Ausdruck dieses Erfolges ist sicherlich der langsame, aber stetige Anstieg des ,impact factor" sowie die, insbesondere in den letzten Jahren, stark anwachsende Zahl der Downloads von in der ZGG publizierten Artikeln. Im Jahr 2011 ging zudem das von der Deutschen Gesellschaft für Geriatrie zunächst in Eigenregie publizierte European Journal of Geriatrics in der ZGG auf, sicherlich im Rückblick eine gute Entscheidung.

Die deutsche Geriatrie braucht aus den verschiedensten Gründen eine Zeitschrift wie die ZGG. Zum einen ist sie die Plattform eines wissenschaftlichen Diskurses, der nicht in englischsprachigen Zeitschriften geführt werden kann, da sich manche Arbeiten auf die strukturellen Besonderheiten der deutschen Geriatrie beziehen, für die auf internationaler Ebene nur wenig Interesse besteht. Gleichwohl sind die entsprechenden Analysen für uns deutsche Geriater von großer Relevanz. Zum anderen können wir auch heute nicht erwarten, dass jeder Kollege für aktuelle Fortbildungsartikel auf die angloamerikanische Literatur zurückgreift. So werden auch in den nächsten Jahren gut konzipierte und redigierte Artikel in deutscher Sprache ihre Berechtigung behalten. Gleichwohl gilt es, in diesem Kontext zu bedenken, dass die $Z G G$ englischsprachige Arti- kel benötigt, da es nur auf diese Weise gelingt, auch außerhalb des deutschsprachigen Raumes wahrgenommen $\mathrm{zu}$ werden und Zitate $\mathrm{zu}$ generieren, die wiederum die Höhe des „impact factor“ bestimmen. Insofern möchten wir hier um Verständnis dafür werben, dass auch zukünftig zahlreiche englischsprachige Artikel in der $Z G G$ erscheinen werden.

In der Ihnen vorliegenden Jubiläumsausgabe haben wir für Sie den Schwerpunkt „Geriatrie: Standards und Perspektiven“" zusammengestellt. Dieser enthält Beiträge zu wichtigen geriatrischen Themen, von denen sich viele auch im Curriculum für die Zusatzweiterbildung Geriatrie finden. Letzteres befindet sich gegenwärtig in Überarbeitung. Die Geriatrie bedarf auch zukünftig einer Weiterentwicklung. $\mathrm{Zu}$ dieser gehört auch eine Vertiefung ihrer Inhalte. Zusammenstellungen wie die vorliegende mögen hier den Weg weisen, wie diese in den nächsten Jahren gelingen kann.

Die einzelnen Beiträge fokussieren daher die Entwicklungen der letzten Jahre in diesen Bereichen. So soll ein Überblick geschaffen werden, um zu erkennen, wo die Hauptaktivitäten in den letzten Jahren lagen, wo sich Wissenschaftler und Arbeitsgruppen besonders eingebracht haben, und welche Themenschwerpunkte für die nächste Zeit auf unserer Agenda stehen müssen.

Natürlich darf ein Resümee zur Geriatrie nicht fehlen, wie es Prof. Gerald Kolb eindrücklich vornimmt, bevor dieses Heft sich einzelnen Themen wie Mobilität, Ernährung, Akutversorgung und Stoffwechselerkrankungen widmet. Weitere aktuelle Beiträge beschäftigen sich mit den Problembereichen Schmerz und Polypharmazie, die uns in der Behand- 
lung unserer geriatrischen Patienten täglich begegnen. Assessment und prognostische Abschätzungen sind ein weiterer wichtiger Bestandteil, wie auch die Weiterentwicklung der Aus-, Fort- und Weiterbildung in der Geriatrie in Deutschland sowie im gesamteuropäischen Kontext.

Der Umfang der Beiträge lässt jeweils nur einen ersten Einblick in die relevanten Themen der Geriatrie zu. Natürlich können in diesem beschränkten Umfang nicht alle Facetten angemessen dargestellt werden, und die Art der Darstellung unterscheidet sich absichtlich von Reviews oder Originalarbeiten. Mit dieser Auswahl sollen die Themen, die die Geriatrie derzeit bewegen, den interessierten Leserinnen und Lesern schmackhaft gemacht werden. Wir werden uns bemühen, viele von ihnen zukünftig in adäquater Weise zu vertiefen. Seien Sie aber auch versichert, dass wir uns über Ihre Einreichungen zu wichtigen Aspekten der Geriatrie freuen werden.

Ihre

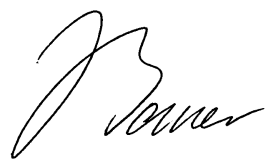

Prof. J.M. Bauer<smiles>C1CCCC1</smiles>

Prof. H.J. Heppner

\section{Korrespondenzadresse}

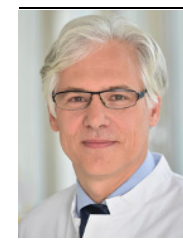

Prof. Dr. med. J. M. Bauer

Geriatrisches Zentrum der Universität Heidelberg,

Agaplesion Bethanien Krankenhaus Heidelberg

Rohrbacher Str. 149,

69126 Heidelberg,

Deutschland

juergen.bauer@

bethanien-heidelberg.de

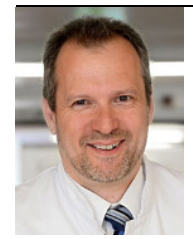

Prof. Dr. med. H. J. Heppner HELIOS Klinikum Schwelm,

Geriatrische Klinik und Tagesklinik, Universität Witten/Herdecke

Dr.-Moeller-Straße 15, 58332 Schwelm, Deutschland Hans.Heppner@uni-wh.de

Interessenkonflikt. J.M. Bauer und H.J. Heppner geben an, dass kein Interessenkonflikt besteht. 